\title{
Prevention of viral induced asthma attacks using inhaled budesonide
}

\author{
G Connett, W Lenney
}

\begin{abstract}
Thirty two preschool children were entered into a double blind, placebo controlled study using intermittent budesonide to treat viral induced wheeze. Active treatment was either $800 \mu \mathrm{g}$ twice a day via a spacer or $1600 \mu \mathrm{g}$ twice a day via a spacer and facemask in those children too young to use a mouthpiece. Treatment was started at the onset of an upper respiratory tract infection and continued for seven days or until symptoms had resolved for $\mathbf{2 4}$ hours. Each child remained in the study until they had completed using one pair of budesonide and placebo inhalers in random order without the need for additional oral prednisolone.
\end{abstract}

Twenty five children completed 28 treatment pairs. All 25 families were asked to express a preference after completing their first treatment pair: 12 preferred budesonide and six preferred placebo; seven had no preference. Symptom scores were compared in 17 treatment pairs that were completed without the need for oral prednisolone. Mean day and night time wheeze in the first week after infection were significantly lower in those receiving budesonide.

Intermittent inhalation of budesonide can modify the severity of wheezing in preschool children developing asthma after viral respiratory infections but improvements were modest with the doses used in this study.

\section{(Arch Dis Child 1993;68:85-7)}

Viral upper respiratory tract infection (URTI) is the most frequent precipitating factor associated with acute childhood wheezing. ${ }^{1}$ In keeping with this, a common pattern of asthma in the preschool age is the child who wheezes only with respiratory infections and is completely well between attacks. Bronchodilator treatment alone is often insufficient and because symptoms are intermittent, continuous prophylactic treatment may seem unjustified. When continuous inhaled steroids are used to treat this pattern of symptoms, the results are often disappointing.

Oral prednisolone is an effective treatment for children presenting with acute wheezing ${ }^{2} 3$ and short courses of inhaled steroids can also
This is a feasible approach. Up to $70 \%$ of children have specific prodromal symptoms for approximately 24 hours before the onset of wheezing. ${ }^{6}$ Commencing medication during this initial prodrome period might successfully decrease or prevent subsequent asthma. This study aimed to prevent viral induced asthma in preschool children by starting inhaled budesonide at the onset of upper respiratory tract symptoms.

\section{Patients and methods}

PATIENTS

Thirty two children aged 1-5 years were recruited. All had been referred to the Royal Alexandra Hospital for Sick Children, Brighton, with acute wheezing and had responded clinically to treatment with bronchodilators. They had consistently wheezed after URTIs and were asymptomatic between attacks. None was receiving prophylactic treatment. All had a history of at least two attacks of wheezing in the six months before recruitment. Children were taught to inhale aerosol medication through a Nebuhaler (Astra). Those too young to master this delivery system were provided with a Nebuhaler modified by the attachment of a Laerdal facemask.

\section{METHODS}

Children were recruited over a six month period (November 1990-April 1991). Parents were given two inhalers (A and $B$ ) to be used consecutively to treat their child's next two respiratory infections. These were identical and contained either budesonide $200 \mu \mathrm{g} /$ actuation or placebo in random order. A terbutaline metered dose inhaler $(250 \mu \mathrm{g} /$ puff $)$ was provided for the treatment of breakthrough symptoms. Parents were told to commence treatment with inhaler $A$ at the onset of upper respiratory symptoms which typically precipitated asthma attacks. Four puffs $(800 \mu \mathrm{g})$ twice a day were used in children using the Nebuhaler alone and eight puffs $(1600 \mu \mathrm{g})$ twice a day in children using the Nebuhaler and facemask. Treatment continued until children were asymptomatic for 24 hours, or until seven days of treatment had been given.

Parents were told to give four to eight puffs (1000-2000 $\mu \mathrm{g})$ of terbutaline every four hours for breakthrough symptoms and to consult their general practitioner as they would do normally. Inhaler B was used to treat the next URTI in exactly the same way. If prednisolone had been prescribed to treat asthma symptoms developing after either URTI, parents were invited to \footnotetext{
modify the severity of asthma attacks. ${ }^{4} \mathrm{~A}$ recent Canadian study has gone one stage further and advocated the use of intermittent steroids as preventative treatment. Oral corticosteroids were used at the onset of upper respiratory symptoms in children whose asthma was predicted by viral infections. Wheezing and the demand for inpatient services were reduced. ${ }^{5}$

Correspondence to: Dr Lenney.

Accepted 3 September 1992

Hospital for

Sick Chidren, 
continue in the study with a second pair of inhalers. Preference for inhaler A or B was ascertained from each set of parents after completing treatment with both inhalers. Preference was analysed by a comparison of proportions. If children received a second treatment pair, preferences after the first pair only were used in this part of the analysis.

Symptom score cards including records of drug usage were kept for two weeks after the onset of symptoms. Upper respiratory symptoms and day and night time cough and wheeze were scored separately on $0-3$ scales $(0=$ no symptoms, $1=$ mild, $2=$ moderate, $3=$ severe). Follow up was arranged every six weeks until children had received treatment with both inhalers. Paired $t$ tests were performed on symptom score data from completed treatment pairs in which prednisolone was not given. Intrasubject comparisons of 16 paired treatments were needed to be $80 \%$ sure of detecting a significant difference in mean first week symptom scores at the $5 \%$ level. This prediction was based on the mean and standard deviation of symptom scores during acute asthma attacks in a previous study. Larger numbers were necessary to detect differences in other outcome variables.

Local ethical committee approval was obtained for the study and parents gave informed written consent.

\section{Results}

Thirty two children entered the study. Seven were withdrawn. Three of these did not have URTIs over 10,13 , and 14 month study periods despite fulfilling the entry criterion of at least two URTIs in the six months before commencing the study. Two were non-compliant with the study protocol and two did not tolerate the Nebuhaler and facemask. In the remaining 25 children, four 'false starts' occurred when parents commenced treatment with an inhaler after mild nasal symptoms that did not develop into an URTI. Treatment was discontinued on each occasion and the same inhaler was used for the next episode. Three episodes of acute asthma occurred in three children before parents had an opportunity to commence treatment. In these children the study inhaler was not started until a subsequent respiratory infection occurred.

Twenty two children completed one treatment pair and three children managed to complete two treatment pairs after receiving prednisolone during their first pair. There were 14 boys and 11 girls. Eleven children between the ages of 1.5 and 2.7 years used the mask and the other 14 used the Nebuhaler alone. The number of colds ranged between two and four in the last six months. Five had a history of bronchiolitis (respiratory syncytial virus negative on each occasion) and 12 had a family history of atopy.

Eighteen of the 25 families expressed a preference after they had completed using the first pair of inhalers. Twelve preferred budesonide and six preferred placebo. This increased preference for budesonide was significant $(\mathrm{p}<0.05)$.

Eleven of the 28 treatment pairs were complicated by the use of prednisolone. This was given for symptoms after one episode in 10 children and one child received prednisolone after both inhalers. Two of these children required hospital admission. In the 10 children who were given prednisolone once, eight had received placebo and two had received budesonide. This difference was not significant when tested for using McNemar's test for paired data (with or without Yates's correction) because of the small numbers available for analysis.

There were 17 treatment pairs for which no intervention with oral steroids was necessary. The mean (SD) number of days on which study medication was given was similar in the two groups: budesonide $5.5(1.4)$ days and placebo $5.9(1.5)$ days. Plots of the mean first week scores, bronchodilator usage, and length of symptoms approximated to normal distributions, supporting the use of parametric statistics to analyse the data. The mean daytime and mean night time wheeze in the first week of the URTI was significantly less in those children who had received budesonide (table 1 ). The mean differences were small and approximated to a normal distribution (figure). There were no significant differences between groups for cough symptoms or bronchodilator usage. Differences for length of symptoms favoured active treatment but numbers were small and liable to a type II error (table 2).

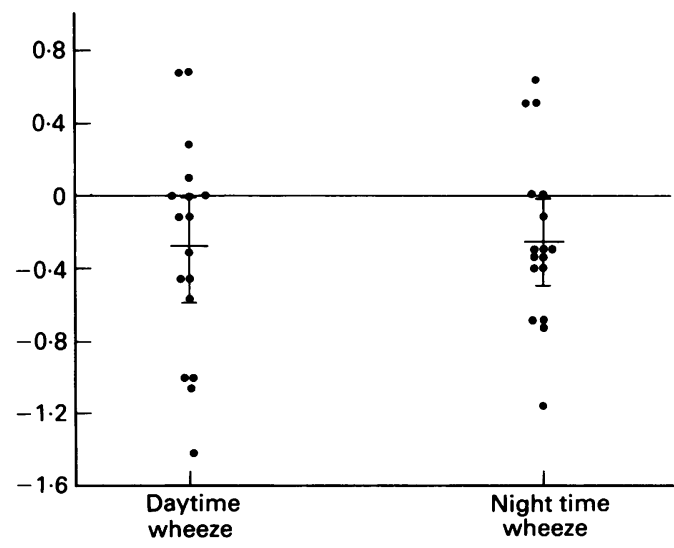

Differences between scores in the 17 treatment pairs not requiring oral prednisolone. Lines indicate mean differences and $95 \%$ confidence intervals.

Table 1 Mean symptom scores in 17 pairs in which treatment with oral steroids did not occur on the first week of the URTI

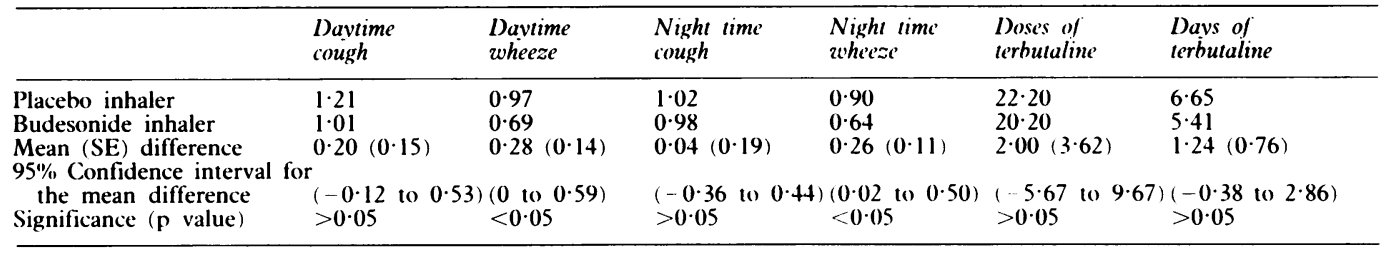


Table 2 Mean length of symptoms in 17 pairs in which treatment with oral steroids did not occur

\begin{tabular}{lllll}
\hline Daytime & $\begin{array}{l}\text { Daytime } \\
\text { cough }\end{array}$ & $\begin{array}{l}\text { Davtime } \\
\text { wheeze }\end{array}$ & $\begin{array}{l}\text { Night time } \\
\text { cough }\end{array}$ & $\begin{array}{l}\text { Night time } \\
\text { wheeze }\end{array}$ \\
\hline Placebo inhaler & $7 \cdot 23$ & 5.47 & 6.23 & $4 \cdot 76$ \\
Budesonide inhaler & 5.82 & $4 \cdot 12$ & $5 \cdot 29$ & 3.47 \\
Mean (SE) difference & $1.41(0.84)$ & $1.35(0.79)$ & $0.94(0.87)$ & $1.29(0.71)$ \\
95\% Confidence interval & $(-0.38$ to $3 \cdot 20)$ & $(-0.32$ to 3.03) & $(-0.91$ to 2.79) & $(-0.20$ to 2.79) \\
Significance (p value) & $>0.05$ & $>0.05$ & $>0.05$ & $>0.05$ \\
\hline
\end{tabular}

\section{Discussion}

The idea of using intermittent inhaled steroids to prevent the development of acute asthma is an attractive one. With the increased awareness that 'asthma' is responsible for many of the respiratory illnesses causing wheeze in childhood, ${ }^{7}$ courses of oral steroids are increasingly given. While short, infrequent dosaging is unlikely to cause significant side effects, the amount of prednisolone needed to treat children with frequent infective exacerbations might adversely affect growth and adrenal function. ${ }^{89}$ Acute behavioural changes after oral prednisolone might limit its usefulness in some children and compliance with continuous inhaled preventative treatment is often poor when symptoms are intermittent.

This study suggests that commencing budesonide at the onset of an URTI reduces the severity of wheezing in children who only have asthma symptoms after upper respiratory infections. Coughing was not eased by treatment. This might have been because it was caused by concomitant viral bronchitis, unresponsive to anti-inflammatory treatment.

Although this study provides statistical evidence for these conclusions, the benefits of treatment were not always clinically obvious and parental preferences were often marginal. Ensuring that parents accurately followed the treatment protocol was time consuming, and necessitated repeated explanations and checks that inhalers were used correctly. In most cases some lower respiratory symptoms were present by 24 to 36 hours after the onset of an URTI. Upper respiratory symptoms were similar in each episode. There were striking intrasubject similarities in the reported patterns of cough and wheeze for each treatment pair, but intersubject illnesses varied widely.

During the study there were fewer documented episodes of URTIs than the number reported in the six months before starting. The mean number of infective exacerbations in the first six months of the study was $2 \cdot 11$ compared with the reported mean of 2.93 over the six months before the study. The difference between the means was 0.82 , with a $95 \%$ confidence interval of 0.288 to $1.34(p<0.001)$. In many cases the first six months of the study included late autumn and winter when colds are more frequent. This suggests that either the frequency of respiratory illness decreased during the study or that parents tended to exaggerated when recollecting their child's symptoms.

The management of intermittent wheeze remains problematic. Complete prevention of asthma symptoms is only conceivable if treatment is given early during upper respiratory symptoms. This is often not achievable because of the time it takes for parents to consult the doctors. Perhaps larger doses of inhaled steroid would be more effective than the doses we have used. The amount of drug reaching the airways using a spacer and facemask is likely to be imprecise and treatment failure was more common when this system was used. This might be due to unresponsiveness to steroids in the younger age group or problems with the delivery system. A study using an alternative spacer and mask has suggested that less than $1 \%$ of delivered doses reach the lungs. ${ }^{10}$ The doses chosen for the study were quite empirical, although budesonide, $200-400 \mu \mathrm{g}$ twice a day through the same delivery system can alleviate chronic persistent asthma in the same age group (unpublished data).

Preschool children with asthma are placing increasing demands on hospital and general practice services. ${ }^{11} 12$ Heterogenous patterns of wheeze in this age group make it difficult to apply the lessons from studies in older asthmatics. ${ }^{13}$ Increasing awareness of the different subgroups of young wheezy children should encourage the further use of novel therapeutic strategies specific to different patterns of symptoms.

We thank Fiona Reed for her statistical advice. GC is supported by the Royal Alexandra Hospital Rocking Horse Appeal.

1 Carlsen KH, Ostravik I, Leegard J, Hoeg H. Respiratory virus infections and aeroallergens in acute bronchial asthma. Arch Dis Child 1984;59:310-5.

2 Storr J, Barrell E, Barry W, Lenney W, Hatcher G. Effect of a single dose of prednisolone in acute childhood asthma. Lancet 1987; i:879-81.

3 Deshpande A, McKenzie SA. Short courses of steroids in home treatment of children with acute asthma. BMJ 1986;293:169-71.

4 Wilson NM, Silverman M. Treatment of acute, episodic asthma in preschool children using intermittent high dose asthma in preschool children using intermittent high dose

5 Brunette MG, Lands L, Thibidou LP. Childhood asthma: prevention of attacks with short-term corticosteroid treatment of upper respiratory tract infection. Pediatrics 1988 81:624-9.

6 Beer S, Laver J, Karpuch J, Chabut S, Aladjem M. Prodromal features of asthma. Arch Dis Child 1987;62 345-8.

7 Hill R, Williams J, Tattersfield A, Britton J. Change in use of asthma as a diagnostic label for wheezing illness in school children. BMF 1989.299.898.

8 Wolthers OD, Pedersen S. Short term linear growth in asthmatic children during treatment with prednisolone. asthmatic children durin

9 Dolan LM, Kesarwala HH, Holroyde JC, Fisher TJ. Short-term, high dose, systemic steroids in children with asthma: the effect on the hypothalamic-pituitary-adrenal axis. F Allergv Clin Immunol 1987;80:81-7.

10 Salmon B, Wilson NM, Silverman $M$. How much aerosol reaches the lungs of whee\%y infants and toddlers. Arch Dis Child 1988;65:401-3.

11 Storr J, Barrell E, Lenney W. Rising asthma admissions and self referral. Arch Dis Child 1988;63:774-9.

12 Anderson HR. Increase in hospital admissions for childhood asthma: trends in referral, severity and readmissions from 1970-1985 in a health region of the United Kingdom. Thorax 1989:44:614-9.

13 Sporik R, Holgate ST, Cogswell JJ. Natural history of asthma in childhood-a birth cohort study. Arch Dis Child 1991; 66: $1050-3$ 